\title{
ON SASAKIAN SUBMANIFOLDS
}

\author{
Dedicated to Professor Shigeo Sasaki on his 60th birthday \\ MiNORU HARADA
}

(Received September 14, 1971; Revised November 29, 1972)

1. Introduction. In his recent papers [4], [5], and [6], K. Ogiue studied positively curved submanifolds of a complex projective space. The purpose of this paper is to study similar problems for submanifolds of a Sasakian space form.

Let $M$ be a $(2 n+1)$-dimensional Sasakian manifold with the structure tensors $\phi, \xi, \eta$, and $g$. Then we have

$$
\begin{gathered}
\phi \xi=0, \quad \eta(\xi)=1, \quad \phi^{2}=-I+\xi \otimes \eta, \\
g(X, \xi)=\eta(X), \quad g(\phi X, \phi Y)=g(X, Y)-\eta(X) \eta(Y), \\
d \eta(X, Y)=g(\dot{\phi} X, Y), \\
\phi X=\nabla_{X} \xi, \quad\left(\nabla_{X} \phi\right) Y=\eta(Y) X-g(X, Y) \xi .
\end{gathered}
$$

By a $\phi$-holomorphic sectional curvature $H(X)$ of $M$ with respect to a unit vector $X$ orthogonal to $\xi$, we mean the sectional curvature $K(X, \phi X)$ spanned by the vectors $X$ and $\phi X$.

A sasakian space form is, by definition, a connected and complete Sasakian manifold of constant $\phi$-holomorphic sectional curvature $C$.

It is known that there are three types of simply connected Sasakian space forms:

1) Elliptic Sasakian space form: $C>-3$; (homeomorphic to a sphere),

2) Parabolic Sasakian space form: $C=-3$; (homeomorphic to a Euclidian space),

3) Hyperbolic Sasakian space form: $C<-3$; (homeomorphic to a real line bundle over a unit disk in $C^{n}$ ).

Simply connected Sasakian space forms are homogeneous contact manifolds which they are regular $[1,8]$.

The author expresses his hearty thanks to Professors K. Ogiue and S. Tanno for their valuable suggestions and encouragement.

2. Sasakian submanifolds. Let $\widetilde{M}$ be a $(2(n+p)+1)$-dimensional Sasakian space form of constant $\phi$-holomorphic sectional curvature $\widetilde{C}$ with structure tensors $(\tilde{\phi}, \tilde{\xi}, \tilde{\eta}, \widetilde{g})$, and let $M$ be a $(2 n+1)$-dimensional differential 
manifold with an almost contact structure $(\phi, \xi, \eta, g)$. We assume that $M$ is immersed in $\tilde{M}$ by $f$ and $f$ satisfies $\tilde{\phi} \cdot f_{*}=f_{*} \cdot \phi, \tilde{\xi}=f_{*} \cdot \xi, \eta=f^{*} \tilde{\eta}$ and $g=f^{*} \widetilde{g}$, where $f^{*}$ denotes the differential of $f$ and $f^{*}$ the dual map of $f_{*}$.

We denote by $\nabla$ (resp. $\tilde{\nabla}$ ) the covariant differentiation with respect to $g$ (resp. $\widetilde{g}$ ). Then the second fundamental form $\alpha$ of the immersion $f$ is given by

$$
\alpha(X, Y)=\tilde{\nabla}_{X} Y-\nabla_{X} Y
$$

We can easily see that $\alpha$ satisfies

$$
\begin{gathered}
\tilde{\phi} \alpha(X, Y)=\alpha(\phi X, Y)=\alpha(X, \phi Y), \\
\alpha(X, \xi)=0 .
\end{gathered}
$$

Let $\nu_{1}, \cdots, \nu_{p}, \tilde{\phi} \nu_{1}, \cdots, \tilde{\phi} \nu_{p}$ be local fields of orthonormal vectors normal to $M$. If we set, for $i=1,2, \cdots, p$,

$$
\begin{aligned}
g\left(A_{i} X, Y\right) & =\widetilde{g}\left(\alpha(X, Y), \nu_{i}\right), \\
g\left(A_{i^{*}} X, Y\right) & =\widetilde{g}\left(\alpha(X, Y), \tilde{\phi} \nu_{i}\right),
\end{aligned}
$$

then, $A_{1}, \cdots, A_{p}, A_{1^{*}}, \cdots, A_{p^{*}}$ are local fields of symmetric linear transformations and they satisfy

$$
\begin{gathered}
A_{i^{*}}=\phi A_{i}, \\
\phi A_{i}=-A_{i} \phi, \\
A_{i} \xi=0 .
\end{gathered}
$$

It is known that $(\phi, \xi, \eta, g)$ is a Sasakian structure on $M$ (Tanno [9]). Hereafter, we therefore call $M$ a Sasakian submanifold of $\widetilde{M}$.

Proposition 2.1 (Tanno [9]). $M$ is a minimal submanifold of $\tilde{M}$.

Proof. It suffices to verify that $\operatorname{tr} A_{i}=\operatorname{tr} A_{i^{*}}=0$. From (2.3) and (2.4), $\operatorname{tr} A_{i^{*}}=0$ is evident, and we have $\phi A_{i} \phi=-\phi^{2} A_{i}=A_{i}-\xi \otimes \eta A_{i}$. Hence we have

$$
\begin{aligned}
\operatorname{tr} A_{i} & =\operatorname{tr}\left(\dot{\phi} A_{i} \phi+\xi \otimes \eta A_{i}\right) \\
& =\operatorname{tr}\left(A_{i} \phi^{2}+\xi \otimes \eta A_{i}\right) \\
& =\operatorname{tr}\left(-A_{i}+2 \xi \otimes \eta A_{i}\right) \\
& =\operatorname{tr}\left(-A_{i}\right),
\end{aligned}
$$

because $\xi \otimes \eta A_{i} X=\eta\left(A_{i} X\right) \xi=g\left(A_{i} X, \xi\right) \xi=g\left(X, A_{i} \xi\right)=0$ by (2.5). q.e.d.

Let $R$ be the curvature tensor field of $M$. Then, the equation of Gauss is 
(2.6)

$$
\begin{aligned}
R(X, Y) Z= & \sum_{i=1}^{P}\left\{-g\left(A_{i} X, Z\right) A_{i} Y+g\left(A_{i} Y, Z\right) A_{i} X\right. \\
& \left.-g\left(\phi A_{i} X, Z\right) \phi A_{i} Y+g\left(\phi A_{i} Y, Z\right) \phi A_{i} X\right\} \\
& +\frac{1}{4}(\widetilde{C}+3)\{g(Y, Z) X-g(X, Z) Y\} \\
& +\frac{1}{4}(\widetilde{C}-1)\{\eta(X) \eta(Z) Y-\eta(Y) \eta(Z) X+g(X, Z) \eta(Y) \xi \\
& -g(Y, Z) \eta(X) \xi-d \eta(X, Z) \phi Y+d \eta(Y, Z) \phi X \\
& -2 d \eta(X, Y) \phi Z\} .
\end{aligned}
$$

Let $S$ and $\rho$ be the Ricci tensor and the scalar curvature of $M$ respectively. Then we have

$$
\begin{aligned}
S(X, Y)= & \frac{1}{2}\{n(\widetilde{C}+3)+\widetilde{C}-1\} g(X, Y)-\frac{1}{2}(n+1)(\widetilde{C}-1) \eta(X) \eta(Y) \\
& -2 \sum_{i=1}^{P} g\left(A_{i} X, A_{i} Y\right)
\end{aligned}
$$

and

$$
\rho=\frac{n}{2}\{(2 n+1)(\widetilde{C}+3)+\widetilde{C}-1\}-2 \operatorname{tr} \sum_{i=1}^{P} A_{i}^{2} .
$$

Let $K(X, Y)$ be the sectional curvature of $M$ determined by orthonormal vectors $X$ and $Y$. Then we have

$$
\begin{aligned}
K(X, Y)= & g(R(X, Y) Y, X) \\
= & \sum_{i=1}^{P}\left\{g\left(A_{i} X, X\right) g\left(A_{i} Y, Y\right)-g\left(A_{i} X, Y\right)^{2}\right. \\
& \left.+g\left(\phi A_{i} X, X\right) g\left(\phi A_{i} Y, Y\right)-g\left(\phi A_{i} X, Y\right)^{2}\right\} \\
& +\frac{1}{4}(\widetilde{C}+3)+\frac{1}{4}(\widetilde{C}-1)\left\{3 g(\phi X, Y)^{2}-\eta(X)^{2}-\eta(Y)^{2}\right\} .
\end{aligned}
$$

In particular, the $\phi$-holomorphic sectional curvature $H(X)$ of $M$ is given by

$$
H(X)=\widetilde{C}-2 \sum_{i=1}^{P}\left\{g\left(A_{i} X, X\right)^{2}+g\left(\phi A_{i} X, X\right)^{2}\right\} .
$$

It is easily seen that $K(\xi, X)=\widetilde{K}(\tilde{\xi}, X)=1$.

\section{Fiberings of Sasakian submanifolds.}

Proposition 3.1. A Sasakian submanifold of a regular Sasakian manifold is also regular. 
Proof. Let $\widetilde{M}$ be a regular Sasakian manifold and $M$ a Sasakian submanifold of $\tilde{M}$. Let $\gamma$ be an integral curve of $\xi$ through a point $P$ of $M$. Then $f(\gamma)$ is an integral curve of $\hat{\xi}=f_{*} \xi$ through $f(P) \in \widetilde{M}$. Assume that $\gamma$ is not regular at $Q \in \gamma(s)$. Let $U_{f(Q)}$ be an arbitrary open neighborhood of $f(Q)$ in $\tilde{M}$. Then, by assumption, $f^{-1}\left(U_{f(Q)}\right)$ is piersed at least twice by $\gamma$. This implies that $U_{f(Q)}$ cannot be regular neighborhood, which is a contradiction.

q.e.d.

By a well known theorem of Boothby-Wang [1], a compact regular Sasakian manifold is a circle bundle over a compact Kaehler manifold. If $M / \xi($ resp. $\tilde{M} / \tilde{\xi})$ denotes the set of orbits of $\xi$ (resp. $\tilde{\xi}$ ), then $M / \xi$ (resp. $\widetilde{M}(\tilde{\xi})$ is a compact Kaehler manifold.

Proposition 3.2. Let $M$ be a compact Sasakian submanifold of a compact regular Sasakian manifold $\widetilde{M}$. Then $M / \xi$ is a compact Kaehler submanifold of $\tilde{M} / \tilde{\xi}$.

Proof. Let $f$ be the immersion of $M$ into $\tilde{M}$, and $\pi: M \rightarrow M / \xi$ (resp. $\tilde{\pi}: \tilde{M} \rightarrow \tilde{M} / \tilde{\xi})$ be the natural projection. Then there exists a mapping $F: M / \xi \rightarrow \widetilde{M} / \widetilde{\xi}$ such that the following diagram is commutative;

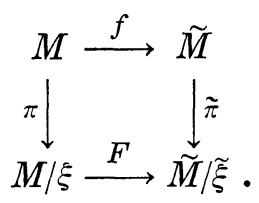

Let $(J, G)(\operatorname{resp} .(\widetilde{J}, \widetilde{G}))$ be the Kaehler structure of $M / \xi(\operatorname{resp} . \tilde{M} / \tilde{\xi})$. Then we have

$$
\begin{aligned}
& (J X)^{*}=\phi X^{*}, \quad G(X, Y)=g\left(X^{*}, Y^{*}\right) \text { for } X, Y \in T(M / \xi), \\
& (\widetilde{J}, \tilde{X})^{*}=\tilde{\phi} \widetilde{X}^{*}, \quad \widetilde{G}(\tilde{X}, \tilde{Y})=\widetilde{g}\left(\widetilde{X}^{*}, \widetilde{Y}^{*}\right) \text { for } \tilde{X}, \tilde{Y} \in T(\tilde{M} / \tilde{\xi}) \text {, }
\end{aligned}
$$

where $*$ denotes the horizontal lift with respect to the connection $\eta$ or $\tilde{\eta}$. For any vector $X$ on $M / \xi$, we have

$$
\begin{aligned}
F_{*}(J X) & =F_{*}\left(\pi_{*} \phi X^{*}\right)=\tilde{\pi}_{*} f_{*}\left(\phi X^{*}\right)=\tilde{\pi}_{*} \phi f_{*} X^{*}=\widetilde{J} \tilde{\pi}_{*} f_{*} X^{*} \\
& =\widetilde{J} F_{*} \pi_{*} X^{*}=\widetilde{J} F_{*}(X),
\end{aligned}
$$

which implies that $F$ is a complex immersion.

On the other hand, we have

$\widetilde{G}\left(F_{*} X, F_{*} Y\right)=\widetilde{g}\left(\left(F_{*} Y\right)^{*},\left(F_{*}, Y\right)^{*}\right)=\widetilde{g}\left(f_{*} X^{*}, f_{*} Y^{*}\right)=g\left(X^{*}, Y^{*}\right)=G(X, Y)$, which implies that $F$ is an isometric immersion.

q.e.d.

Remark. Proposition 3.2 was proved in [2] for hypersurfaces.

Let $R^{\prime}$ be a curvature tensor field of $M / \xi$. Then we have [3] 


$$
\begin{aligned}
\left(R^{\prime}(X, Y) Z\right)^{*}= & -\phi^{2} R\left(X^{*}, Y^{*}\right) Z^{*}-\frac{1}{2} \eta\left(\left[Y^{*}, Z^{*}\right]\right) \phi X^{*} \\
& +\frac{1}{2} \eta\left(\left[X^{*}, Z^{*}\right]\right) \phi Y^{*}+\eta\left(\left[X^{*} \cdot Y^{*}\right]\right) \phi Z^{*} .
\end{aligned}
$$

Let $K^{\prime}(X, Y)$ be the sectional curvature of $M / \xi$ determined by orthonormal vectors $X$ and $Y$. Then we have

$$
K^{\prime}(X, Y)=K\left(X^{*}, Y^{*}\right)+3 g\left(X^{*}, \phi Y^{*}\right)^{2} .
$$

The holomorphic sectional curvature $H^{\prime}(X)$ of $M / \xi$ determined by $X$ is given by

$$
H^{\prime}(X)=H\left(X^{*}\right)+3 \text {. }
$$

Let $S^{\prime}$ be the Ricci tensor of $M / \xi$. Then we have

$$
S^{\prime}(X, Y)=S\left(X^{*}, Y^{*}\right)-g\left(R\left(\xi, X^{*}\right) Y^{*}, \xi\right)+3 g\left(X^{*}, Y^{*}\right) .
$$

The scalar curvature $\rho^{\prime}$ of $M / \xi$ is given by

$$
\rho^{\prime}=\rho+2 n \text {. }
$$

4. Main results. Throughout this section, we confine our attention to compact Sasakian submanifolds of a simply connected elliptic Sasakian space form.

Let $\widetilde{M}$ be a simply connected elliptic Sasakian space form of constant $\phi$-holomorphic sectional curvature $\widetilde{C}(\widetilde{C}>-3)$ and $M$ be a compact Sasakian submanifold of $\widetilde{M}$. Then $\widetilde{M} / \widetilde{\xi}$ is a complex projective space of constant holomorphic sectional curvature $\widetilde{C}+3$ by (3.5).

TheoREM 4.1. Let $M$ be a compact Sasakian submanifold of codimension 2 imbedded (resp. immersed) in a simply connected elliptic Sasakian space form of constant $\phi$-holomorphic sectional curvature $\widetilde{C}$. If $\operatorname{dim} M \geqq 5$ (resp. $\operatorname{dim} M \geqq 9$ ) and if the sectional curvature $K$ of $M$ satisfies $K(X, Y)+$ $3 g(\phi X, Y)^{2}>0$ for each pair of orthonormal vectors $X$ and $Y$, then $M$ is totally geodesic.

Proof. By Proposition 3.2, $M / \xi$ is a compact Kaehler hypersurface imbedded (resp. immersed) in a complex projective space. Our assumption, together with (3.4), implies that every sectional curvature of $M / \xi$ is positive. Hence, by Theorem 3.3 in [4], (resp. Theorem in [5]) $M / \xi$ is a totally geodesic submanifold of codimension 2 of the complex projective space so that $H^{\prime}=\widetilde{C}+3$. This, together with (3.5), implies $H=\widetilde{C}$. Therefore, by $(2,10)$, we have $A_{1}=0$, that is, $M$ is totally geodesic. q.e.d.

THEOREM 4.2. Let $M$ be a $(2 n+1)$-dimensional compact Sasakian 
submanifold immersed in a simply connected elliptic Sasakian space form of constant $\phi$-holomorpic sectional curvature $\widetilde{C}$ of dimension $2(n+p)+1$. If every $\phi$-holomorphic sectional curvature of $M$ is greater than $\widetilde{C}-$ $((n+2) / 2(n+2 p))(\widetilde{C}+3)$, then, $M$ is totally geodesic.

Proof. $M / \xi$ is an $n$-dimensional compact Kaehler submanifold immersed in a complex projective space of constant holomorphic sectional curvature $\widetilde{C}+3$ of dimension $n+p$. By assumption and (3.5), we have

$$
H^{\prime}>(\widetilde{C}+3)\left(1-\frac{n+2}{2(n+2 p)}\right) \text {. }
$$

By virtue of Theorem in [6], $M / \xi$ is totally geodesic. By the argument similar to Theorem 4.1, $M$ is totally geodesic.

q.e.d.

The same argument as Theorem 4.2, combined with Theorem in [10] implies the following.

THEOREM 4.3. Let $M$ be a 5-dimensional compact Sasakian submanifold immersed in an 11-dimensional simply connected elliptic Sasakian space form of constant $\phi$-holomorphic sectional curvature $\widetilde{C}$. If every $\phi$ holomorphic sectional curvature of $M$ is greater than $(2 / 3) \widetilde{C}-1$, then $M$ is totally geodesic.

THEOREM 4.4. Let $M$ be a $(2 n+1)$-dimensional compact Sasakian submanifold immersed in a simply connected elliptic Sasakian space form of constant $\phi$-holomorphic sectional curvature $\widetilde{C}$. If every Ricci curvature of $M$ is greater than $(n / 2)(\widetilde{C}+3)-2$, then $M$ is totally geodesic.

Proof. For a unit vector $X$ in $M / \xi$, we have from (3.6) that

$$
S^{\prime}(X, X)=S\left(X^{*}, X^{*}\right)+2 \text {. }
$$

This, together with our assumption, implies $S^{\prime}(X, X)>(n / 2)(\widetilde{C}+3)$. Hence, by virtue of Theorem 1 in [6], $M / \xi$ is a totally geodesic submanifold of the complex projective space. By the argument similar to Theorem 4.1, $M$ is totally geodesic.

q.e.d.

TheOREM 4.5. Let $M$ be a $(2 n+1)$-dimensional compact Sasakian submanifold of codimension 2 immbedded in a simply connected elliptic Sasakian space form of constant $\phi$-holomorphic sectional curvature $\widetilde{C}$. If the scalar curvature of $M$ is greater than $(\widetilde{C}+3) n^{2}-2 n$ almost everywhere on $M$, then, $M$ is totally geodesic.

Proof. From (3.7), we have $\rho^{\prime}>(\widetilde{C}+3) n^{2}$, which together with Corollary 2.2 in [4], implies that $M$ is totally geodesic. q.e.d. 


\section{BIBRIOGRAPHY}

[1] W. M. Boothby and H. C. Wang, On contact manifolds, Ann. of Math. 68 (1958) 721734 .

[2] K. Kenmotsu, Invariant submanifolds in a Sasakian manifold, Tôhoku Math. J., 21 (1969) 495-500.

[3] K. OGIUE, On fiberings of almost contact manifolds, Kôdai Math. Sem. Rep., 17 (1965) 53-62.

[4] - Differential geometry of algebraic manifolds, Differential Geometry in honor of Professor Kentaro Yano (1972), 355-372.

[5] - Positively curved complex hypersurface immersed in a complex projective space, to appear.

[6] - Positively curved complex submanifolds immersed in a complex projective space I, II, to appear.

[7] S. SASAKI, Almost contact manifolds, Lecture Note, Tôhoku Univ.

[8] S. TANNo, The automorphism groups of almost contact Riemannian manifolds, Tôhoku Math. J., 21 (1969) 21-38.

[9] - Isometric immersions of Sasakian manifolds in sphere, Kôdai Math. Sem. Rep., 21 (1969) 448-458.

[10] - 2-dimensional complex submanifolds immersed in complex projective spaces, to appear.

TOKYo GaKUgei University 
